\title{
Research Paper \\ The Effect of 14 Days of Coenzyme Q10 Supplementation on Muscle Damage and Fatigue Indices Following a Bout Exhausting Exercise Activity in Passive Men
}

\author{
*Tohid Khanvari ${ }^{1} \odot$, Faramarz Sardari² $\odot$, Babak Rezaei ${ }^{1} \odot$ \\ 1. Department of Exercise Physiology, Faculty of Physical Education and Sport Sciences, University of Tabriz, Tabriz, Iran. \\ 2. Department of Exercise Physiology and Corrective Exercises, Faculty of Sport Sciences, Urumia University, Urumia, Iran.
}

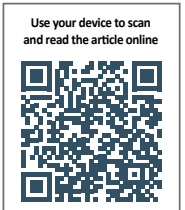

Cittation: Khanvari T, Sardari F, Rezaei B. [The Effect of 14 Days of Coenzyme Q10 Supplementation on Muscle Damage and Fatigue Indices Following a Bout Exhausting Exercise Activity in Passive Men (Persian)]. Journal of Arak University of Medical Sciences (JAMS). 2020; 23(3):386-397. https://doi.org/10.32598/JAMS.23.3.3619.1

Key words:

Exhaustive aerobic activity, Coenzyme Q10 supplementation, Cortisol, Creatine Kinase (CK), Inactive men

\section{ABSTRACT}

Background and Aim Exercise aerobic is associated with increased creatine kinase and blood lactate immediately after exercise, leading to increased muscle damage and undesirable changes in many cellular markers including serum creatine kinase. In such situations, consuming nutrients and supplements such as coenzyme Q10 may prevent metabolic stress damage by increasing buffering power. However, comprehensive studies have not been performed on the effects of this nutrient on the index of muscle injury and fatigue caused by exercise.

Methods \& Materials For this purpose, 20 inactive volunteer men were randomly divided into two groups of 10-person Coenzyme Q10 supplement $(2.5 \mathrm{mg} / \mathrm{kg}$ body weight) and quasi-drug $(2.5 \mathrm{mg} / \mathrm{kg}$ body weight Dextrose). All subjects participated in the Bruce test exercise contract after 14 days of supplementation. Blood sampling was performed in four stages including baseline, after supplementation, immediately after exercise and two hours after exercise. Creatine kinase, lactate and cortisol indices of both groups were measured during these four stages. Data were analyzed by means of standard deviation and repeated measures ANOVA, Bonferroni post hoc and Independent T-test using SPSS V. 17 at the significant level of 0.05 .

Ethical Considerations This article has been approved by the ethics committee of Tabriz School of Medical Sciences with the ethics code IRCT 201203104663N8.

Results The results showed that 14 days of Coenzyme Q10 supplementation had a significant effect on cortisol level $(P<0.05)$. In addition, one session of exhausting aerobic activity increased creatinine kinase and lactate $(P<0.05)$. On the other hand, creatinine kinase did not differ significantly after exercise $(P>0.05)$.

Conclusion According to the results of the present study, 14-day supplementation of Coenzyme Q10 may reduce the cellular damage induced by exhaustive aerobic activity in inactive men and prevent an increase in blood lactate levels.

\section{Extended Abstract}

\section{Introduction}

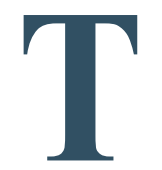

oday, aerobic exercise has become a necessity for health, weight management, and prevention of metabolic diseases [1, 2]. These activities may lead to muscle and tissue damage, facilitate the oxidation of membrane fatty acids, and initiate a series of destructive reactions leading to cell death [3]. In such cases, the use of nutritional supplements and antioxidants is necessary to prevent injuries caused by irregular and intense sports activities. One of these supplements, whose effects have been reported in studies as an antioxidant and anti-fatigue agent, is coenzyme Q10.

\section{* Corresponding Author:}

\section{Tohid Khanvari, PhD.}

Address: Department of Exercise Physiology, Faculty of Physical Education and Sport Sciences, University of Tabriz, Tabriz, Iran.

Tel: +98 (936) 9945857

E-mail: khanvari10@gmail.com 
However, the results of various studies showed that the effects of Q10 on the indicators of fatigue and muscle injuries are contradictory $[9,19]$. Therefore, it is necessary to determine the antioxidant effects of coenzyme Q10 supplementation on injuries caused by exercise [9]. Therefore, the aim of this study was to evaluate the effect of 14 days of coenzyme Q10 supplementation on muscle injury and fatigue indices following a period of strenuous exercise in inactive men.

\section{Materials and Methods}

In a semi-empirical double-blind study, consisting of two groups of complementary and placebo recipients, with repeated measurements (four blood draws), 20 inactive men (Mean age $23 \pm 2$ years and Mean weight $74 \pm 2.01 \mathrm{~kg}$ ), randomly divided into two homogenized groups receiving coenzyme Q10 supplement ( $2.5 \mathrm{mg} / \mathrm{kg}$ body weight, per day) and placebo (dextrose, the same amount of supplement was added during the supplementation period).

Their aerobic capacity was determined using Bruce test on a treadmill. One week later, to homogenize and determine the baseline values of the desired parameters (cortisol, creatine kinase and lactate), before starting the fourteen-day supplement, the first blood samples were taken from the right antecubital vein of all subjects in the amount of five $\mathrm{ml}$. After completing the supplementation period (14 days) and before performing aerobic activity, a second blood sample was taken.

After confirming the natural distribution (KolmogorovSmirnov and Shapiro-Wilk tests) and the homogeneity of the data obtained in the first stage (independent t-test), the research hypotheses were evaluated with repeated measures Analysis of Variance (ANOVA), Bonferroni post 17 at a significance level of 0.05 . In addition, the effect size of each of the interfering factors was determined using omega squared.

\section{Results}

The results showed that coenzyme Q10 supplementation had no significant effect on basal cortisol. In other words, coenzyme Q10 supplementation could not significantly alter basal blood cortisol. However, the increase in cortisol after debilitating aerobic activity was not significant in the coenzyme Q10 supplement group. But in the placebo group, cortisol was significantly increased after debilitating aerobic activity. Cortisol levels fell below baseline 2 hours after exercise. In other words, coenzyme Q10 supplementation can reduce the significant increase in changes in serum cortisol (stress hormone) after debilitating aerobic activity.
On the other hand, the results of ANOVA of cell damage index indicated that loading of coenzyme Q10 supplementation had no effect on serum total creatine kinase. In other words, coenzyme Q10 supplementation could not significantly alter basal blood creatine kinase. On the other hand, the results of ANOVA related to changes in blood lactate indicated that taking coenzyme Q10 supplementation could not cause a significant change in basal blood lactate. However, the increase in lactate after debilitating exercise was significantly lower than in the placebo group. In other words, the effect of the measurement steps on the incidence of changes in blood lactate was greater than the group differences. The results of independent t-test showed that there was a significant difference between the lactate levels of the supplement and placebo groups after exercise.

\section{Discussion}

The results of the present study showed that basal coenzyme Q10 supplementation had no effect on serum total creatine kinase. The possible mechanism of action of coenzyme Q10 as an antioxidant in reducing creatine kinase was probably that coenzyme Q10 reduced peroxidation of membrane fats and reduced damage to phospholipid membranes by removing free bases and increasing the body's antioxidant capacity, and thereby prevented the leakage and penetration of this intracellular enzyme into extracellular fluids $[17,18]$. On the other hand, it was shown that basal coenzyme Q10 supplementation had no effect on blood lactate.

The results of the present study on the increase in plasma lactate levels after debilitating aerobic activity were consistent with the results of the research of Sachek et al. (2003) [21]. In his study of healthy young and old men, Sachek showed that blood lactate increased significantly immediately after 45 minutes of running on the negative slope of the treadmill with $75 \%$ of maximum oxygen consumption; This rate was significant in the group of old people compared to the group of young men [21]. In addition, in the present study, coenzyme Q10 supplementation after aerobic exercise significantly reduced plasma lactate in the supplement group compared to placebo. Probably the reason was the increase in plasma coenzyme Q10 and the strengthening of mitochondrial coenzyme and activation of the aerobic metabolic pathway, which limits lactate production by accelerating the consumption of fatty acids and the production of adenosine triphosphate [14]. 


\section{Ethical Considerations}

Compliance with ethical guidelines

This study was approved by the Ethics Committee of Tabriz University (Code: IRCT201203104663N).

\section{Funding}

The present paper was extracted from the MSc. thesis of the first author, Department of Exercise Physiology, Faculty of Physical Education and Sport Sciences, University of Tabriz.

\section{Authors' contributions}

Methodology, sampling: Faramarz Sardari; Data analysis: Tohid Khanvari; Conceptualization, writing - review \& editing: All authors.

\section{Conflicts of interest}

The authors declared no conflict of interest.

\section{Acknowledgements}

The authors would like to thank the University of Urmia, especially the Faculty of Physical Education and Sports Sciences, the esteemed professors, who helped us in presenting this research. 


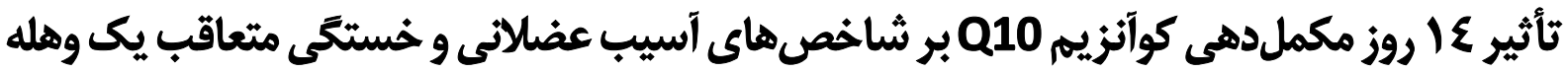

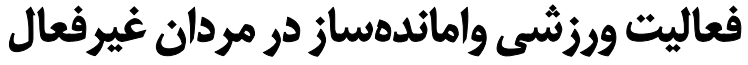

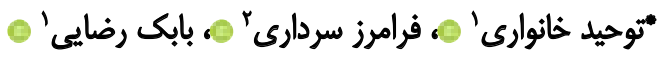 \\ I. كروه فيزيولورى ورزشى، دانشكده تربيت بدنى و علوم ورزشى، دانشكاه تبريز، تبريز، ايران.

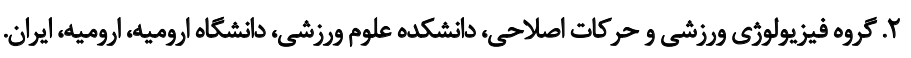

\begin{abstract}
جيكيد

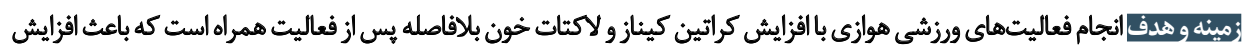

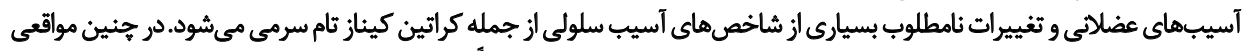

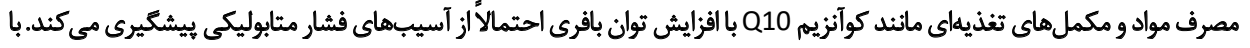

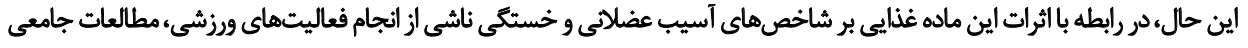
صورت نكرفتئه است.

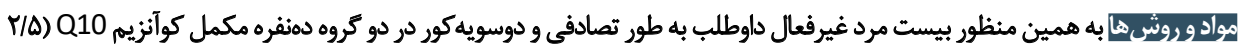

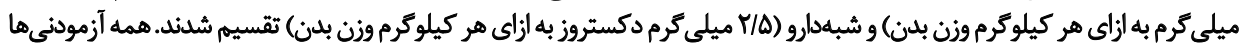

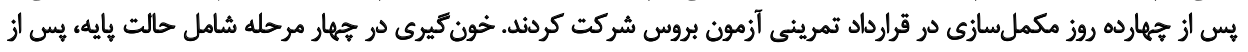

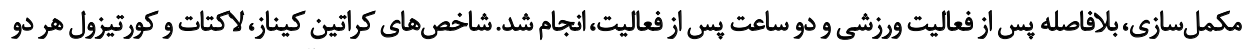

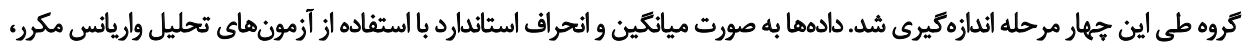

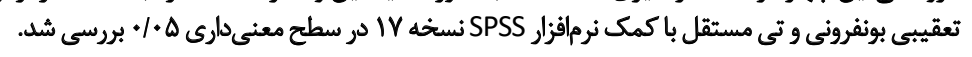

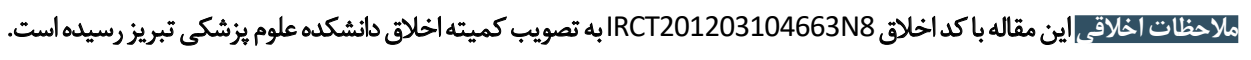

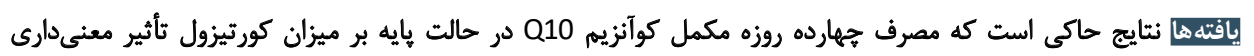

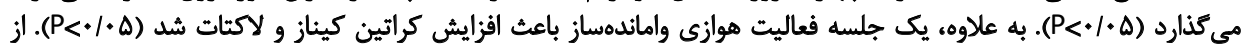

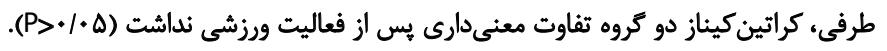

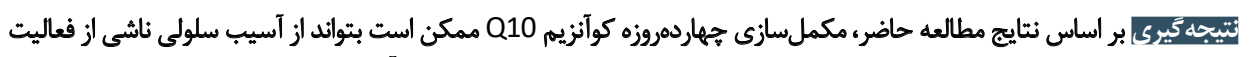

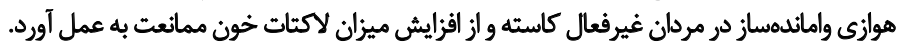

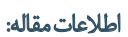

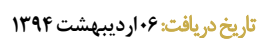

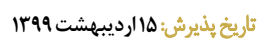

افزايش معنى دارى نشان داد [†] :نتايج تحقيق سوميداو وممكاران

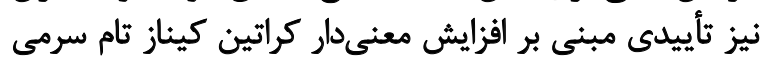

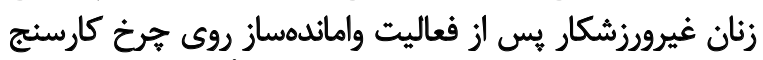

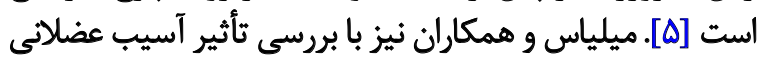

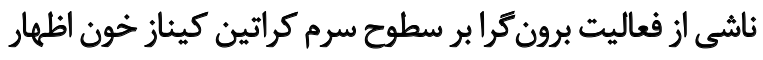

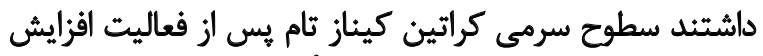

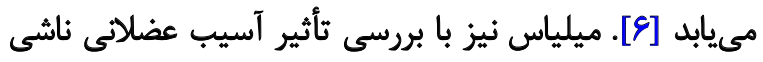

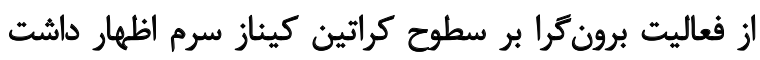

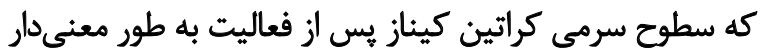

افزايش مى يابد [عاع).

همجنين، عبدالناصر با بررسى اثرات فعاليت واماندهساز بر

dato

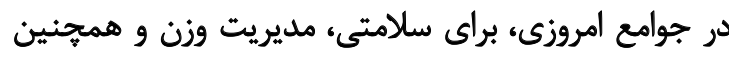

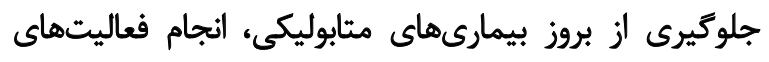

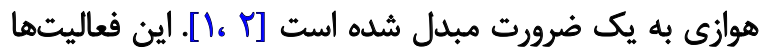

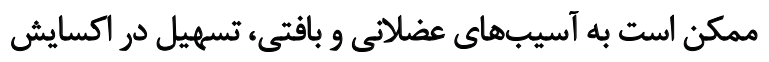

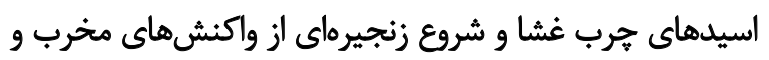

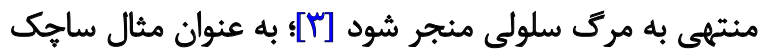

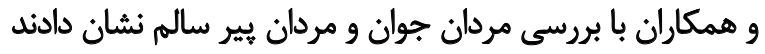

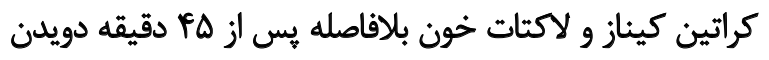

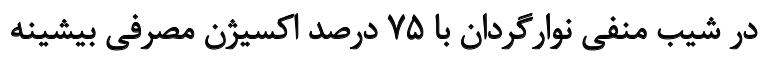

: توحيد خانداه مسئول:

توحيد خانوارى نو نويند

نشائى: تبريز، دانشعاه تبريز، دانشكده تربيت بدنى و وعلوم ورزشي، كروه فيزيولوزئى ورزشى. تلفن: يست الكترونيكي: khanvari10@gmail.com 
شاخصهاى يُيكرسنجى (قد، توده بدنى، درصد جربيى) و سابقه

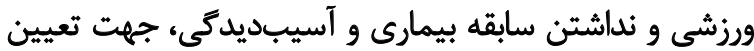
نمونهاي همكن انتخاب شدند.

افراد داوطلب در يك ماه قبل از تحقيق به طور سرخود يا

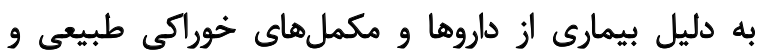

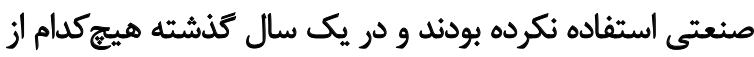

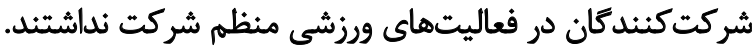

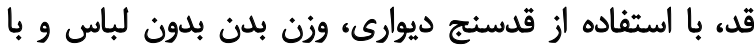

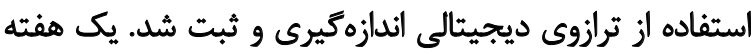

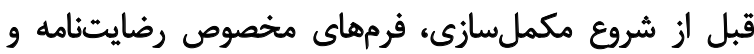

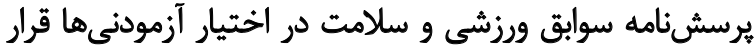

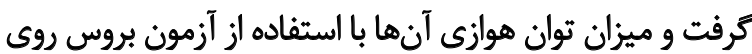
نواركردان تكنوجيم تعيين شد.

يك هفته بعد از تعيين توان هوازى، اولين نمونههاى خونى

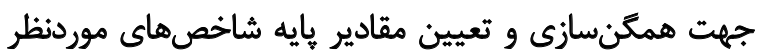

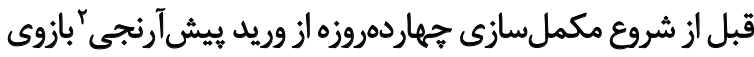

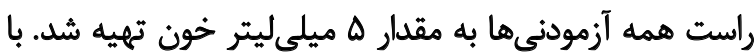

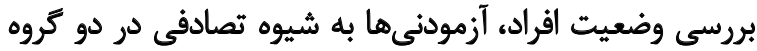

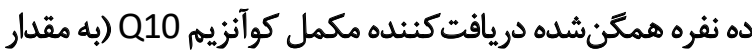

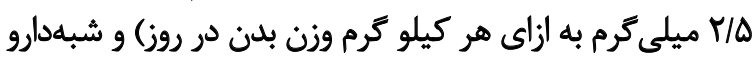

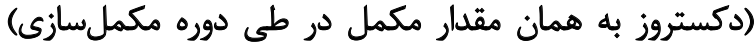

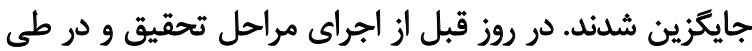

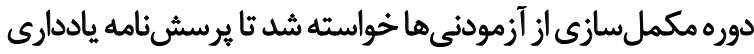

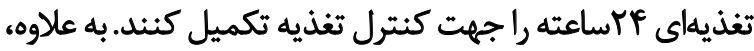

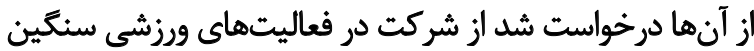

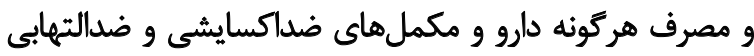

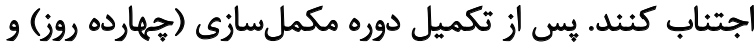

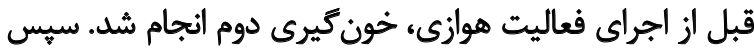

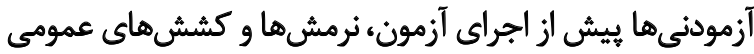

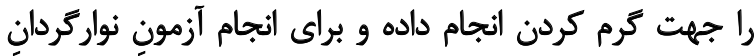

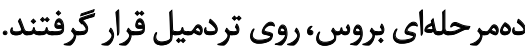

با بررسى وضعيت سلولهاي خونى افراد، بيست نفر به به شيوه

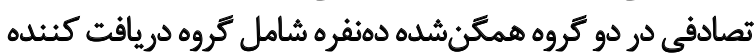

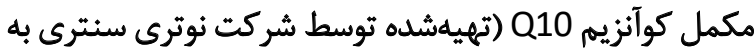

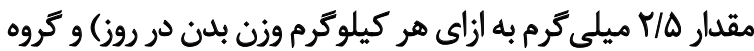

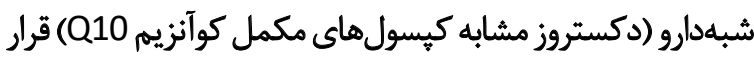

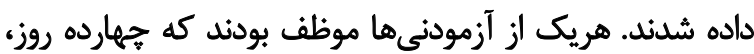

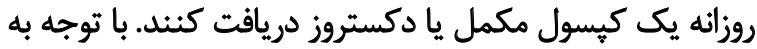

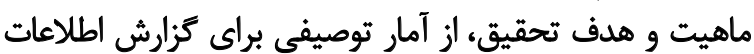

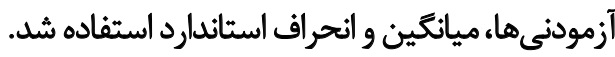

2. Antecubital vien
روى برخى متغيرهاى فيزيولوزيكى در بسكتباليستها تزارش

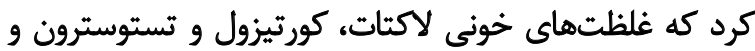

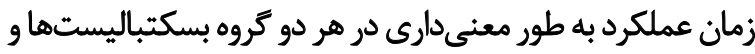

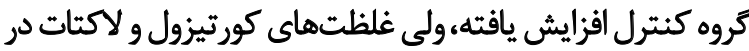

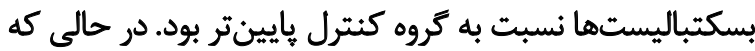

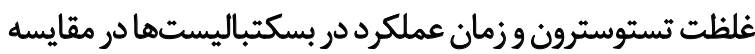

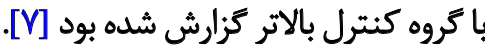

در هنين مواقعى براى جلوكيرى از آسيبهاى ناشى از

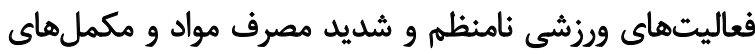

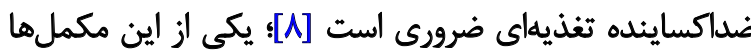

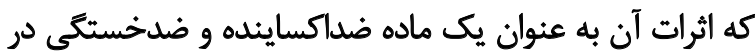

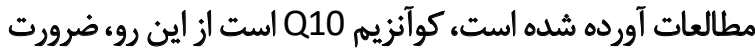

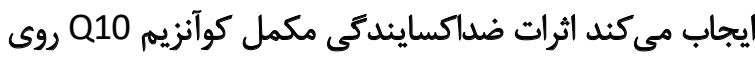

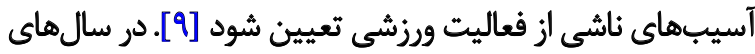

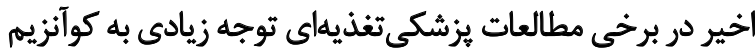

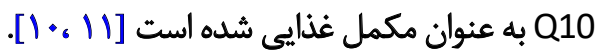
از طرفى، زوليانى و همكاران با مطالعه افراد تمريننكرده

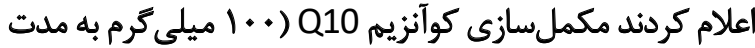

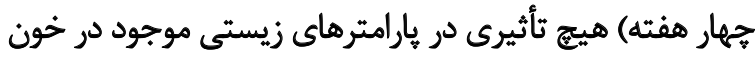

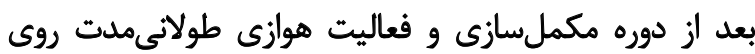

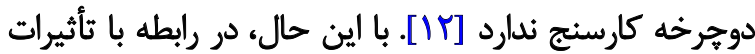

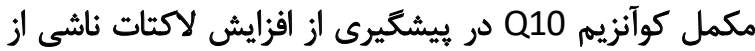

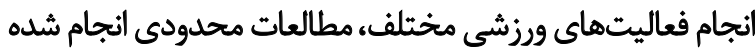

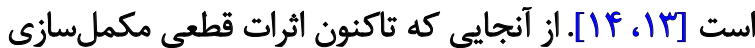

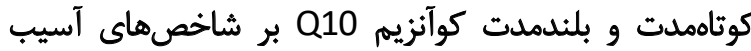

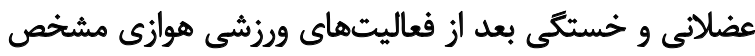

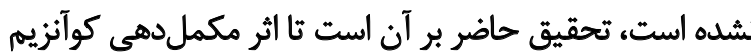

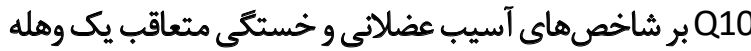
فعاليت ورزشى واماندهاز در مردان غيرفعال را بررسى كند.

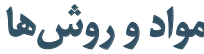

اين تحقيق از نوع نيمهتجربى دو كروهى دوسويهكور'

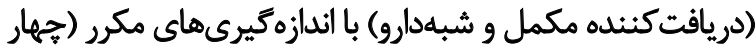

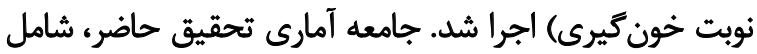

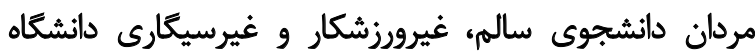

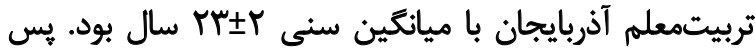

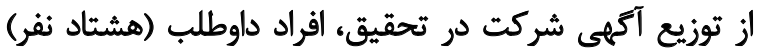

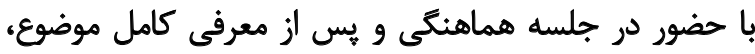

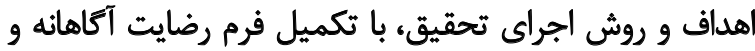

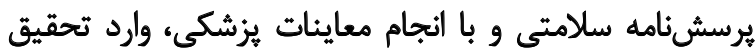

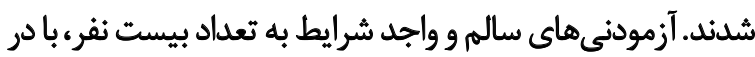

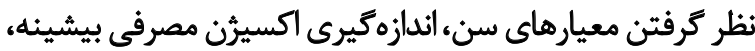

1. Double blind 
جدول ا. كروهها و مراحل اندازه كيرى

\begin{tabular}{|c|c|c|c|c|}
\hline بعد از فيريت ب ساعت & خون ازيري بلافاصله بعد & مكمل خون كيرى بق بعل از فوروه & خون كيرى عا روز قبل از & كروهها و مراحل اندازهكيرى \\
\hline (·ن (نفر) & (· انفر) & (1) (نفر) & (1. (انفر) & كروه دريافتكثنده هكمل \\
\hline (نفر) & (•ا (نفر) & (i (نقر) & (· (انثر) & كروه دريافت كثنده شبهدارو \\
\hline
\end{tabular}

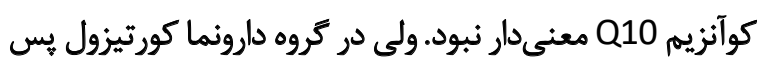

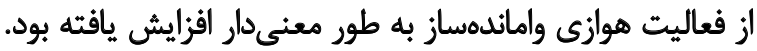

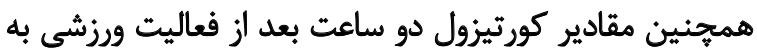

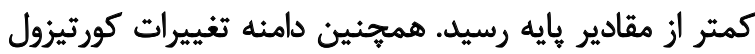

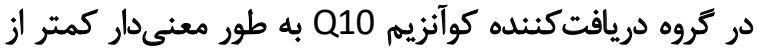

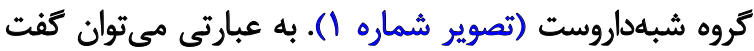

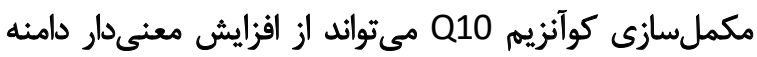

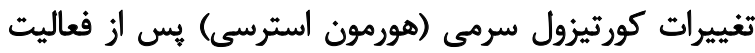

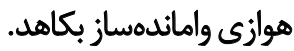

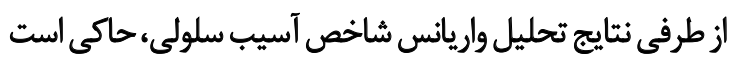

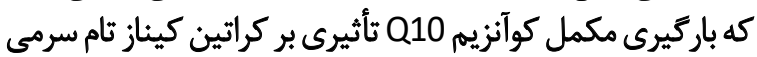

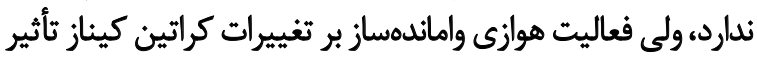

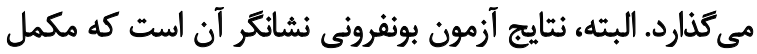

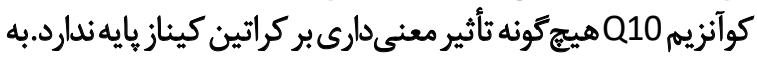

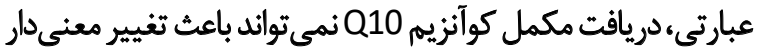

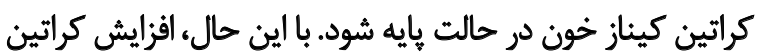

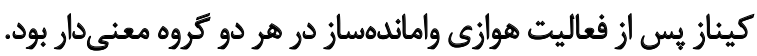

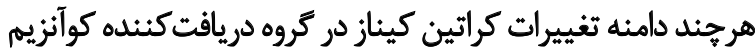

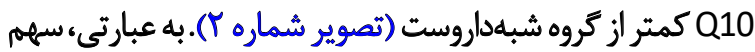

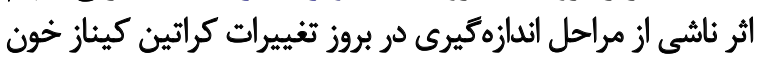
نسبت به تفاوتهاي گروهى بيشتر است.

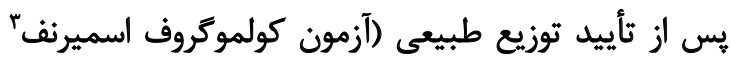

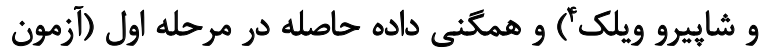

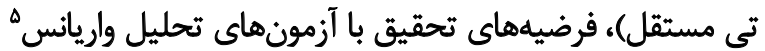

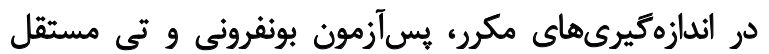

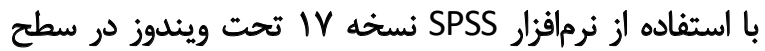

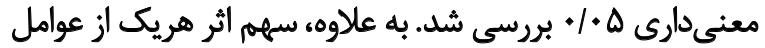

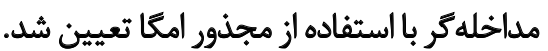

ياقتهها

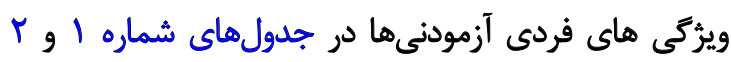

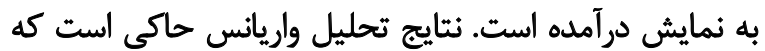

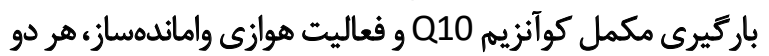

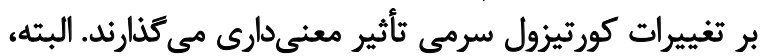

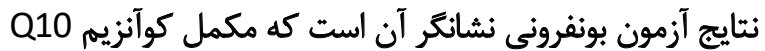

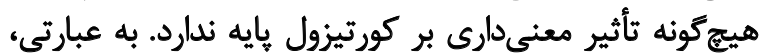

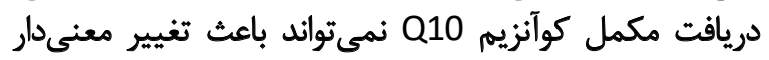

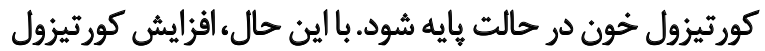

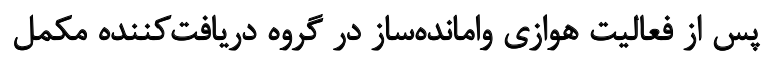

3. Kolmogorov-Smirnov

4. Shapiro-Wilk

5. Analysis of Variance (ANOVA)

جدول ب. نتايج آزمون تى مستقل براى مقايسه متغيرها در كروه مكمل و دارونما

\begin{tabular}{|c|c|c|}
\hline ميانغينين +انحراف استأيدارد & كرو & مشَحصات فردى \\
\hline $\begin{array}{l}r \in / A \pm r / * f \\
r / r^{\prime} \pm r / M\end{array}$ & هكمل & وزن (كيلوكرم) \\
\hline 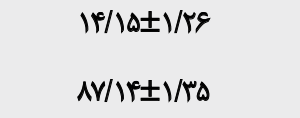 & مكمل & درصد هربى (I/) \\
\hline $\begin{array}{l}\text { NTHE./QT } \\
\text { QRTE/// }\end{array}$ & دكمل & سن (سال) \\
\hline
\end{tabular}




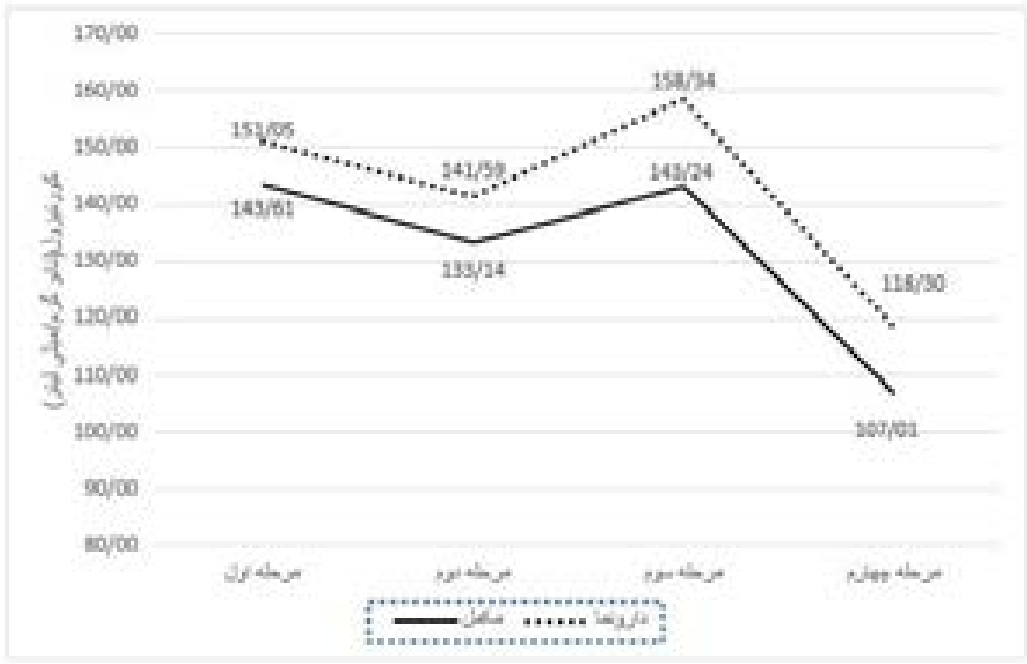

تصوير ا. تغييرات كورتيزول سرم كروهاى مكمل و شبهدارو يس از مكملسازى، فعاليت هوازى و دو ساعت يس از فعاليت هوازى

تفاوت معنى داري وجود دارد (تصوير شماره بّ). بنابراين، فرضيه

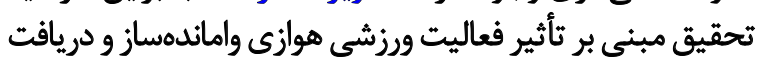

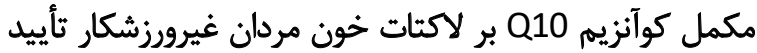

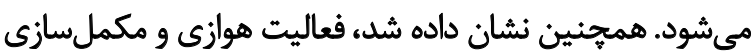

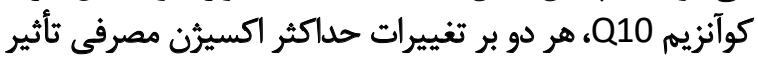

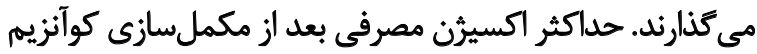

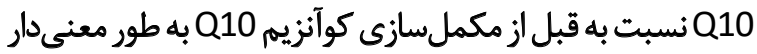

افزايش يافته است (تصوير شماره F).

بحث

يافتههاى تحقيق حاضر حاكى از آن است كه مكملسازى
نتايج تحليل واريانس مربوط به تغييرات لاكتات خون حاكى

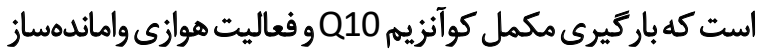

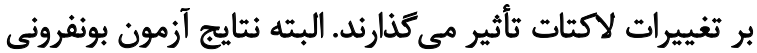

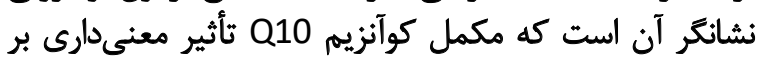

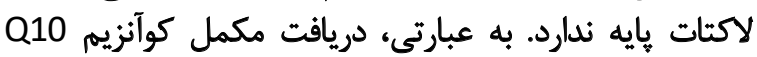

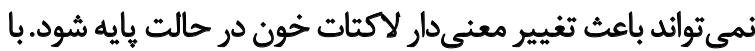

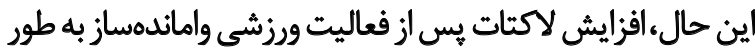

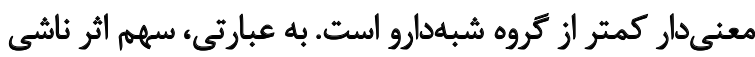

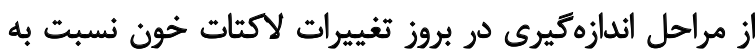

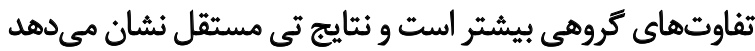

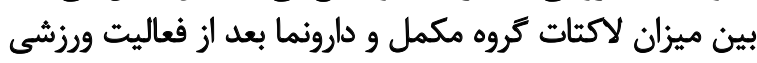
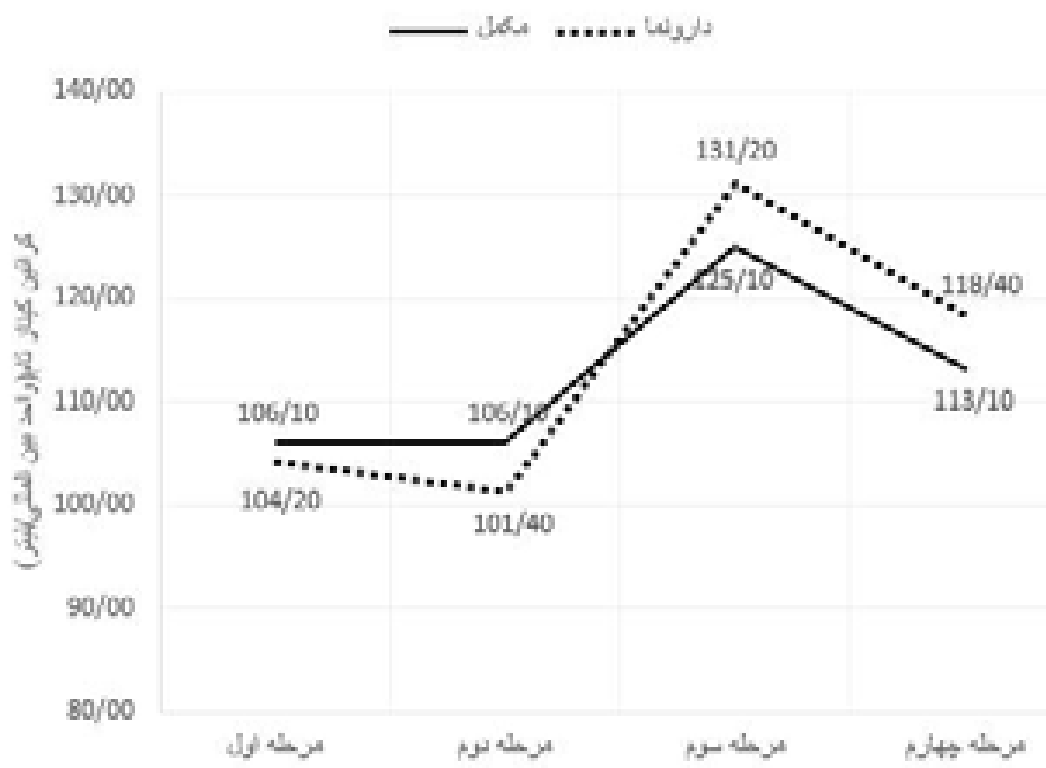


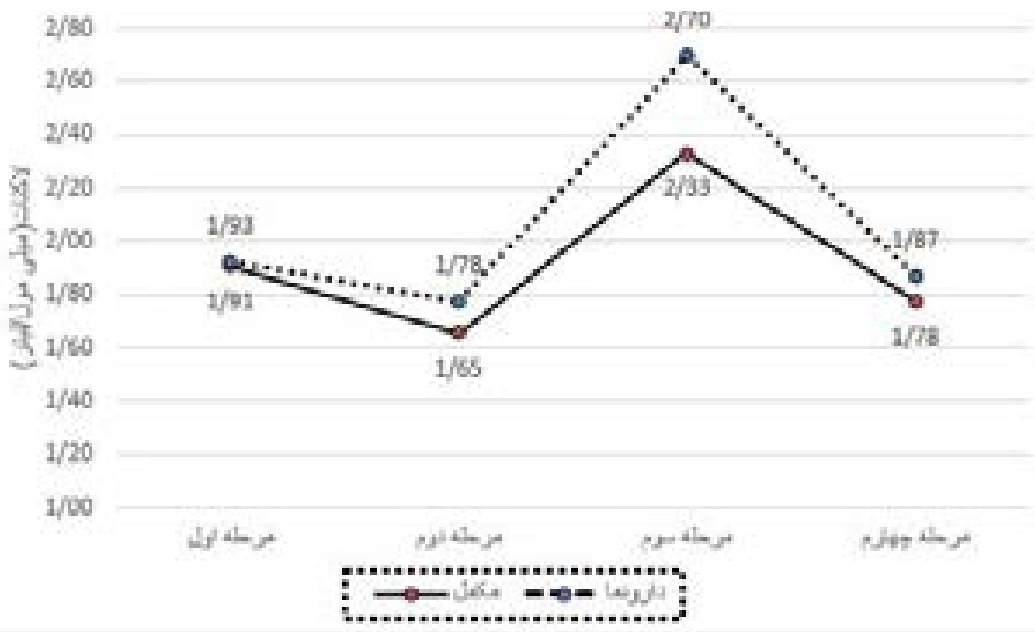

تصوير ب. تغييرات لاكتات هيلاسماى كروههاى مكمل و شبهدارو يس از مكملسازى، فعاليت هوازى و دو ساعت يس از فعاليت هوازى

با كاهش مان ازاى هر كيلوكرم وزن بلن طى سه ماه در ميمونها

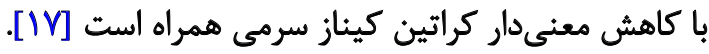
يافته تحقيق حاضر حاكي است كه مكملسازى كوآنزيم

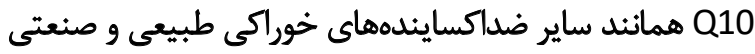

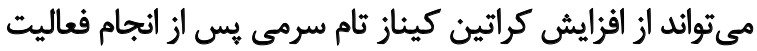
هوازى بكاهد. سازوكار احتمالى تأثير كوآنزيم

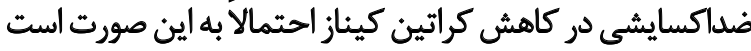

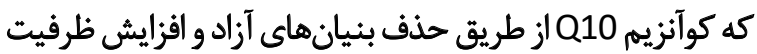

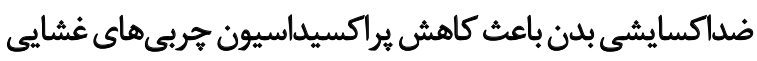

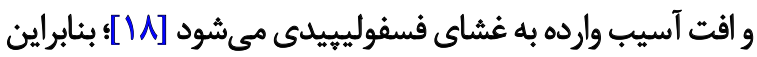

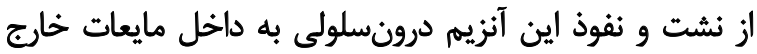

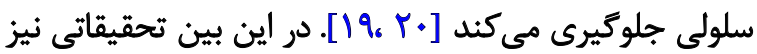
وجود داشته است كه تغييرات اين شاخص مكئ بسيار اندك بوده؛

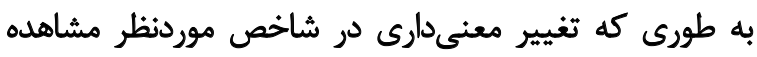

كوآنزيم Q10 در حالت يايه تأثيرى بر كراتين كيناز تام

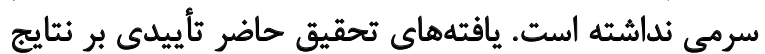

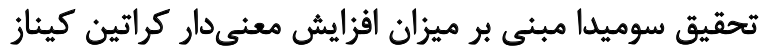

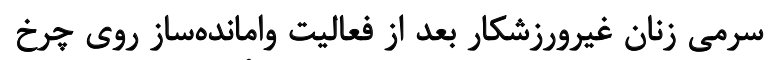

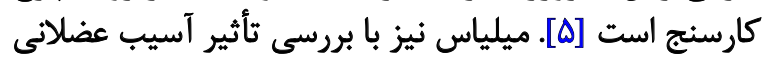

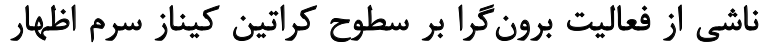

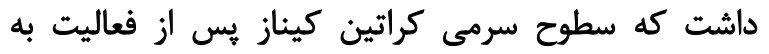

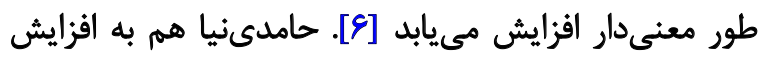

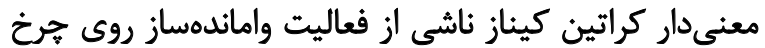

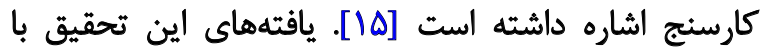

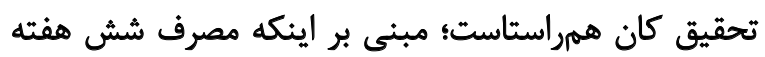

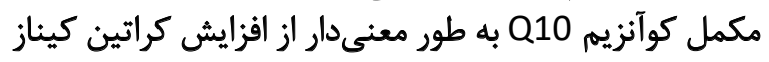

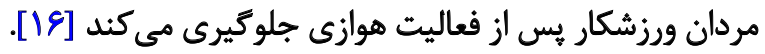

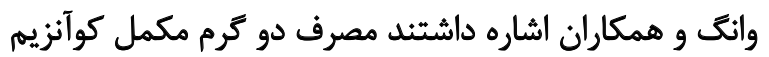

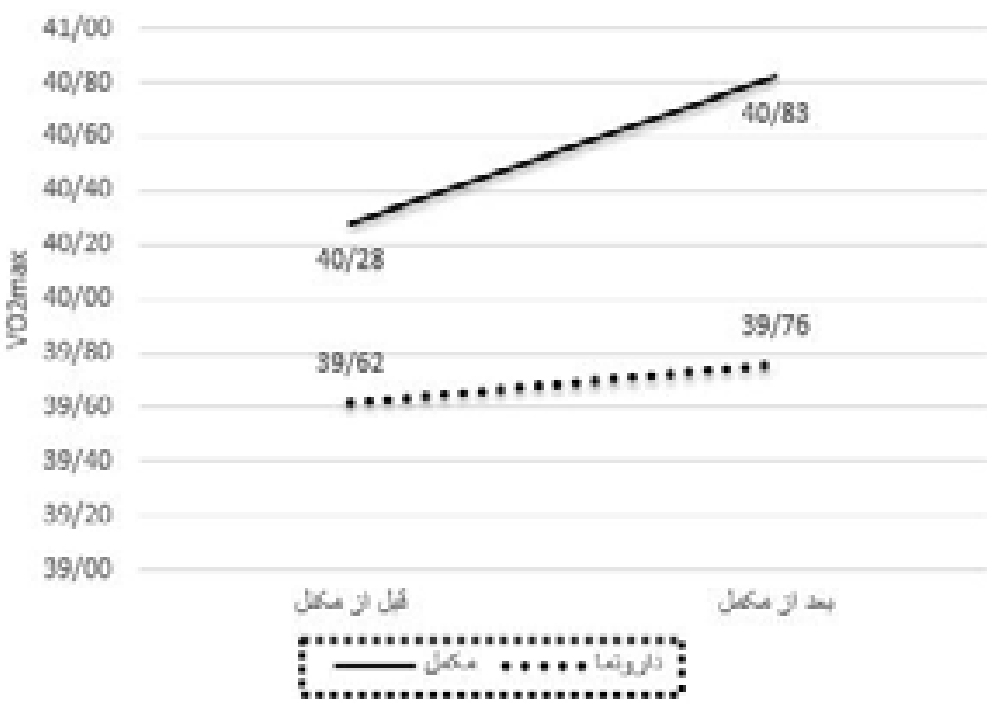

تصوير F. تغييرات VO²max كروههاى مكمل و شبهدارو يس از مكمل سازي، فعاليت هوازى و دو ساعت هس از فعاليت هوازي 
كردند افزايش معنىدارى در غلظتهاى كورتيزول، اسيد

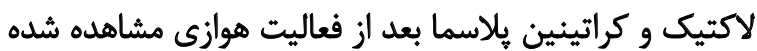

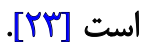

همجئين، عبدالناصر با بررسى اثرات فعاليت واماندهساز روى برى برئي

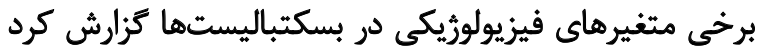

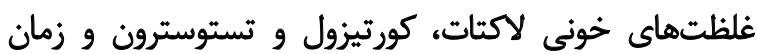

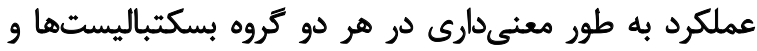

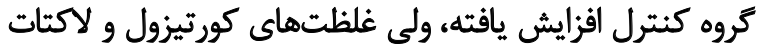

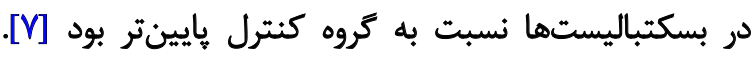

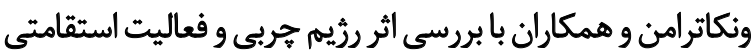

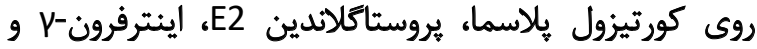

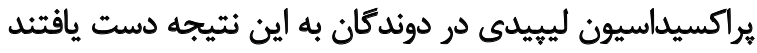

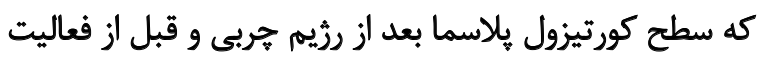

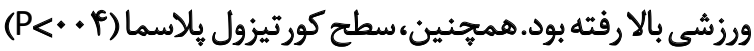

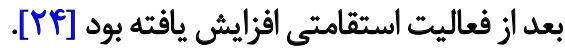

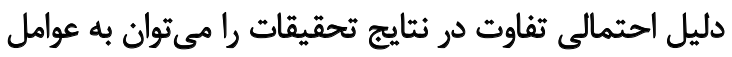

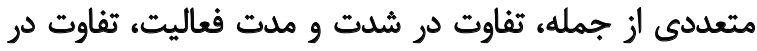

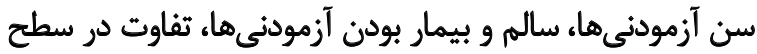

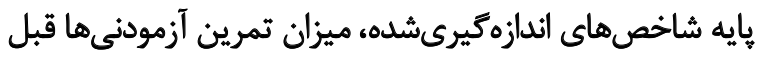

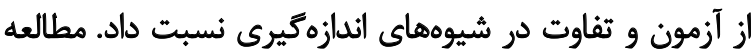

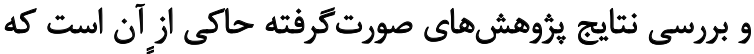

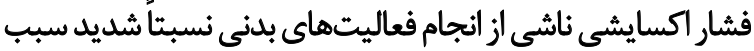

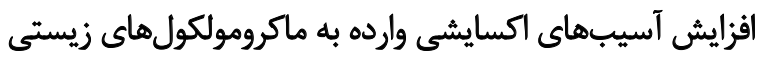

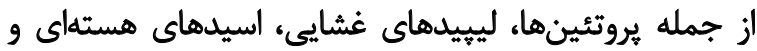

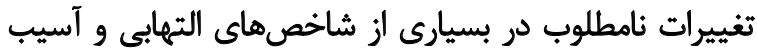

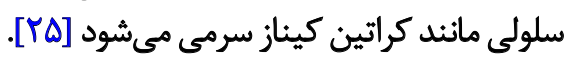

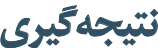

يكى از راههاى مقابله با اثرات نامطلوب فشار اكسايشى ناشى از

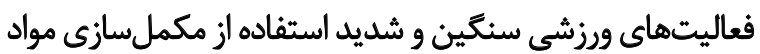

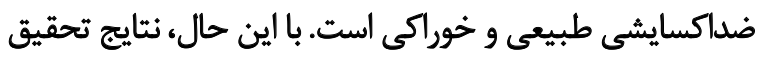

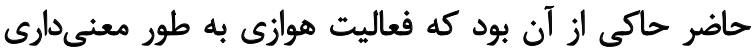

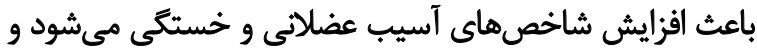

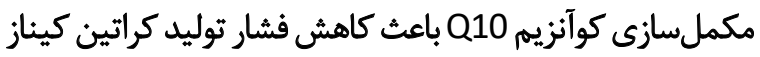

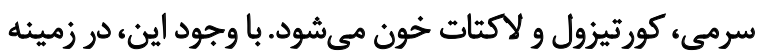

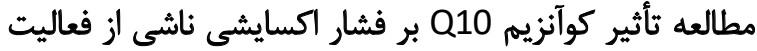
هوازى تحقيقات بيشترى نياز است. بر فثاريم

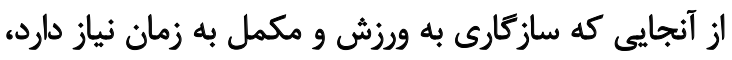

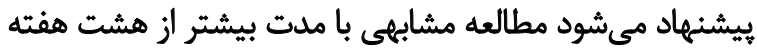

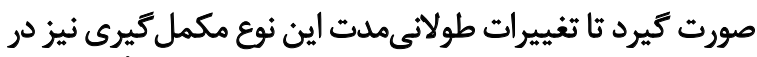

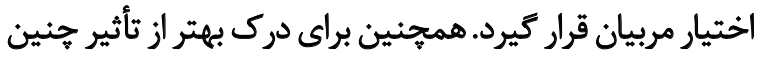

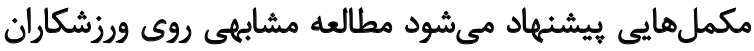

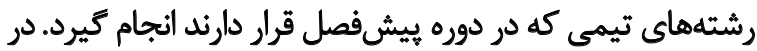

نشده است. از جمله اين تحقيقات مىتوان به تحقيق زوليانى

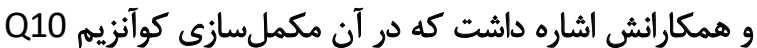

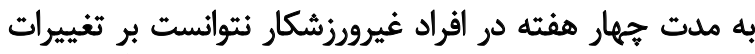

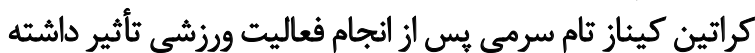

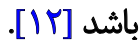

كان و همكاران نيز با مطالعه موشهاي صحرايى دريافتند

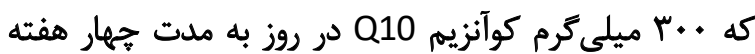

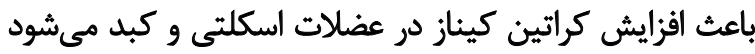

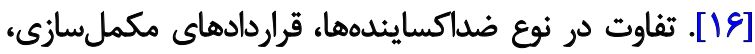

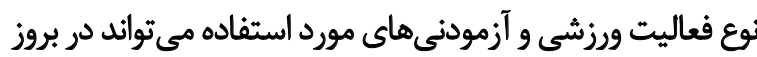

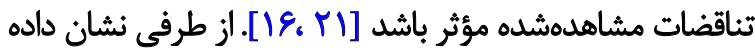

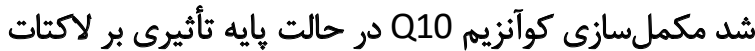
خون نداشته است.

نتايج تحقيق حاضر مبنى بر افزايش ميزان لاكتات يلاسما پِّيس

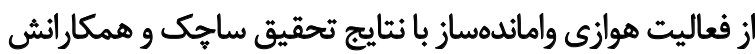

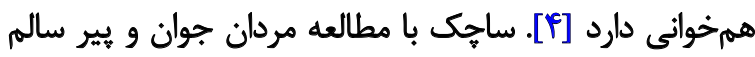

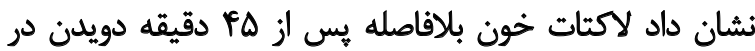

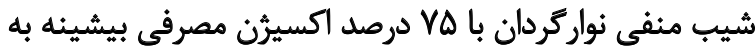

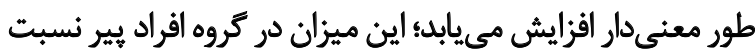

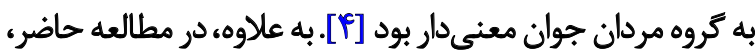

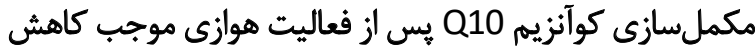

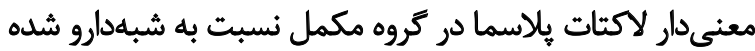

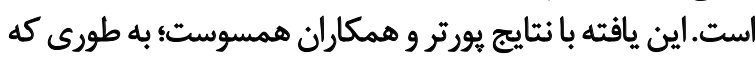

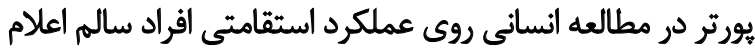

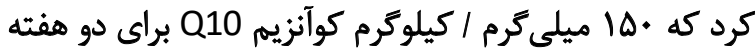

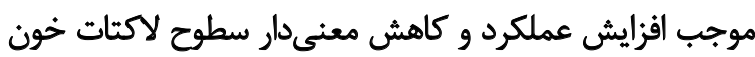

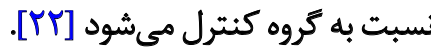

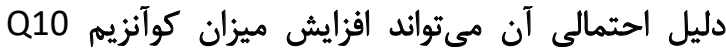

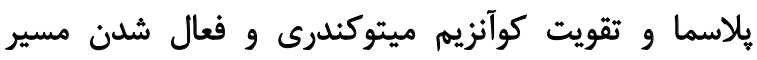

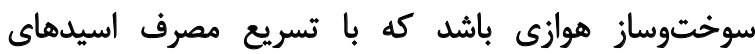

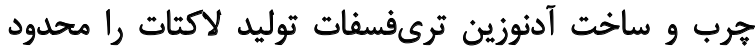

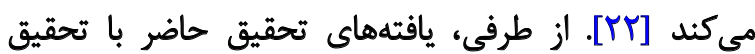

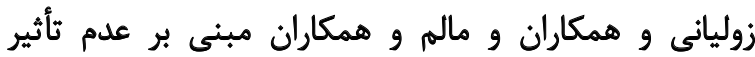

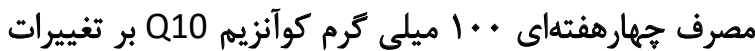

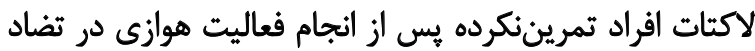

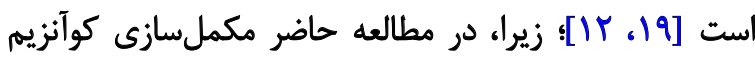

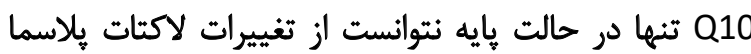

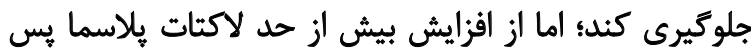

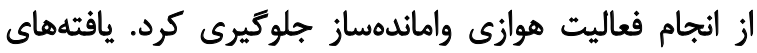

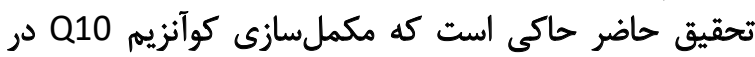

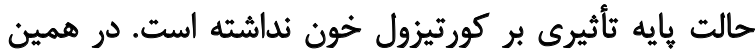

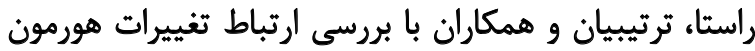

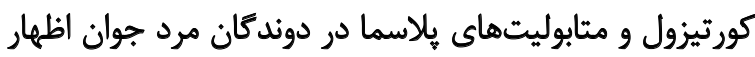


نهايت با توجه به نتايج مطالعه حاضر مربيان ميتوانند از اين نوع

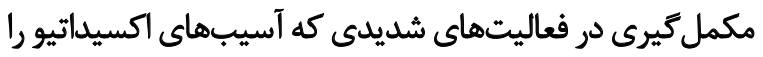
به ورزشكار اعمال مي كند استفاده كنيند. ملاحظات اخلاقى

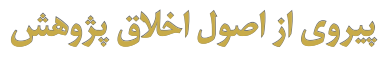
اين مقاله با كد اخلاق RCT201203104663N8 ابه تصويب كميته اخلاق دانشكده علوم يزشكى تبريز رسيده است.

$$
\text { Ullos co }
$$

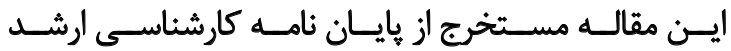

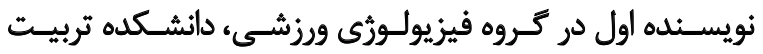

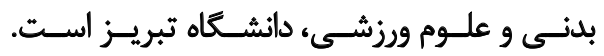

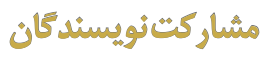

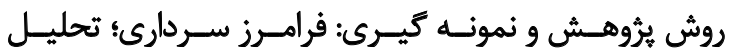

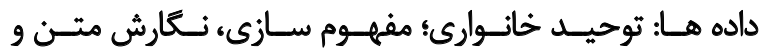

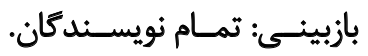

$$
\text { عition }
$$

نويسندكان مقاله هيجّونه تعارضى در منافع اعلام نكردند.

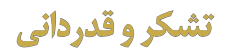

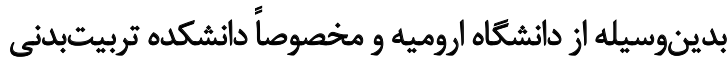

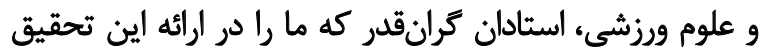
يارى كردند تقدير و تشكر مي كنيه. 


\section{References}

[1] Barutcu A, Taylor S, McLeod CJ, Witcomb GL, James LJ. Planned aerobic exercise increases energy intake at the preceding meal. Med Sci Sports Exerc. 2020; 52(4):968-75. [DOI:10.1249/ MSS.0000000000002199]

[2] Seals DR, Nagy EE, Moreau KL. Aerobic exercise training and vascular function with ageing in healthy men and women. J Physiol. 2019; 597(19):4901-14. [DOI:10.1113/JP277764] [PMID]

[3] Balcı SS, Pepe H, Güney S, Özer Ç, Revan S. Effects of gender, endurance training and acute exhaustive exercise on oxidative stress in the heart and skeletal muscle of the rat. Chin J Physiol. 2012;55(4):23644. [DOI: 10.4077/CJP.2012.BAA021]

[4] Sacheck JM, Milbury PE, Cannon JG, Roubenoff R, Blumberg JB. Effect of vitamin $E$ and eccentric exercise on selected biomarkers of oxidative stress in young and elderly men. Free Radic Biol Med. 2003; 34(12):1575-88. [DOI:10.1016/S0891-5849(03)00187-4]

[5] Sumida S, Doi T, Sakurai M, Yoshioka Y, Okamura K. Effect of a single bout of exercise and $\beta$-carotene supplementation on the urinary excretion of 8-hydroxy-deoxyguanosine in humans. Free Radic Res. 1997; 27(6):607-18. [DOI:10.3109/10715769709097864] [PMID]

[6] Milias GA, Nomikos T, Fragopoulou E, Athanasopoulos S, Antonopoulou $S$. Effects of eccentric exercise-induced muscle injury on blood levels of Platelet Activating Factor (PAF) and other inflammatory markers. Eur J Appl Physiol. 2005; 95(5-6):504-13. [DOI:10.1007/ s00421-005-0031-6] [PMID]

[7] Ghobadi A. Comparison of stress among athlete and aon athlete male students in islamic azad university tehran jonob and shahre-rey branches. Procedia- Soc Behav Sci. 30:(2011):694-7. [DOI: 10.1016/j.sbspro.2011.10.134]

[8] Stajer V, Vranes M, Ostojic SM. Correlation between biomarkers of creatine metabolism and serum indicators of peripheral muscle fatigue during exhaustive exercise in active men. Res Sports Med. 2020; 28(1):147-54. [DOI:10.1080/15438627.2018.1502185] [PMID]

[9] Ochoa JJ, Quiles JL, López-Frías M, Huertas JR, Mataix J. Effect of lifelong coenzyme Q10 supplementation on age-related oxidative stress and mitochondrial function in liver and skeletal muscle of rats fed on a Polyunsaturated Fatty Acid (PUFA)-rich diet. J Gerontol A Biol Sci Med Sci. 2007; 62(11):1211-8. [DOI:10.1093/ gerona/62.11.1211] [PMID]

[10] Cooke M, losia M, Buford T, Shelmadine B, Hudson G, Kerksick C, et al. Effects of acute and 14-day coenzyme Q10 supplementation on exercise performance in both trained and untrained individuals. J Int Soc Sports Nutr. 2008; 5(1):8. [DOI:10.1186/1550-27835-8] [PMID] [PMCID]

[11] Kon M, Tanabe K, Akimoto T, Kimura F, Tanimura Y, Shimizu K, et al. Reducing exercise-induced muscular injury in kendo athletes with supplementation of coenzyme Q 10. Br J Nutr. 2008; 100(4):903-9. [DOI:10.1017/S0007114508926544] [PMID]

[12] Zuliani U, Bonetti A, Campana M, Cerioli G, Solito F, Novarini A. The influence of ubiquinone (Co Q10) on the metabolic response to work. J Sports Med Phys Fitness. 1989; 29(1):57-62. [PMID]

[13] Catarina MQ, Salvatore DM, Michio H. Human coenzyme Q10 deficiency. Neurochem Res. 2007; 32(4-5):723-7. [DOI:10.1007/s11064006-9190-z] [PMID] [PMCID]

[14] Kocharian A. Coenzyme Q10 improves diastolic function in children with idiopathic dilated cardiomyopathy. Cardiol young. 2009; 19(5): 501-6. [DOI:10.1017/S1047951109990795] [PMID]
[15] Hamedinia MR, Gaeini AA. The combined effect of aerobic exercise and vitamins on oxidative stress at rest and after exhausting exercise in student-athletes. Olympic Quart. 2004; 12(3):73-81. http://ensani. ir/file/download/article/20120326111507-1130-92.pdf

[16] Kon M, Kimura F, Akimoto T, Tanabe K, Murase Y, Ikemune S, et al. Effect of Coenzyme Q10 supplementation on exercise-induced muscular injury of rats. Exerc Immunol Rev. 2007; 13:76-88. [PMID]

[17] Wang, Jimmy. Self emulsifying compositions for delivering lipophilic coenzyme Q10 and other dietary ingredients. U.S. Patent Application No. 11/217,867. [Internet]. [Updated 2006 Mar 10] https:// patents.google.com/patent/US20060051462A1/en

[18] Yuvaraj S, Premkumar VG, Vijayasarathy K, Gangadaran SG, Sachdanandam P. Effect of coenzyme Q10, riboflavin and niacin in tomoxifen-treated postmenopausal breast cancer patiente with special reference to lipids and lipoproteins. Cli Bioch. 2007; 40(9-10):623-8. [DOI:10.1016/j.clinbiochem.2007.02.003] [PMID]

[19] Malm C, Svensson M, Ekblom B, and Sjodin B. Effect of ubiquinine-10 supplementation and high intensity training on physical performance in human. Acta Physiol Scand. 1997; 161(3):379-84. [DOI:10.1046/j.1365-201X.1997.00198.x] [PMID]

[20] DiMauro S, Quinzii CM, Hirano M. Mutations in coenzyme Q 10 biosynthetic genes. J Clin Invest. 2007; 117(3):587-9. [DOI:10.1172/ JCI31423] [PMID] [PMCID]

[21] Modi K, Santani DD, Goyal RK, Bhatt PA. Effect of coenzyme Q10 on catalase activity and other antioxidant parameters in streptozotocin-induced diabetic rats. Biolo Tra Elem Res. 2006; 109(1):25-33. [DOI:10.1385/BTER:109:1:025]

[22] Porter DA, Costill DL, Zachwieja JJ, Krzeminski K, Fink WJ, Wagner $E$, et al. The effect of oral coenzyme Q10 on the exercise tolerance of middle-aged, untrained men. Int J Sports Med. 1995; 16(7):421-7. [DOI:10.1055/s-2007-973031] [PMID]

[23] Tartibian B, Yaghoobnezhad F, Saboory E. The response of serum cortisol and lipid profile to a moderately intensive aerobic exercise in non-active middle-aged men. Urmia Med J. 2013; 24.6 (): 393-404. [PMID]

[24] Venkatraman JT, Feng X, Pendergast D. Effects of dietary fat and endurance exercise on plasma cortisol, prostaglandin E2, interferon- $\gamma$ and lipid peroxides in runners. J Am Coll Nutr. 2001; 20(5):529-36. [D OI:10.1080/07315724.2001.10719062] [PMID]

[25] Macdonald J, Galley HF, Webster NR. Oxidative stress and gene expression in sepsis. Br J Anaesth. 2003; 90(2):221-32 [DOI:10.1093/ bja/aeg034] [PMID] 
This Page Intentionally Left Blank 